\title{
CHARGE TRANSFER DYNAMICS IN ATOM-METAL SURFACE COLLISIONS
}

\author{
M. Wiertel, R. Taranko and E. Taranko \\ Institute of Physics, M. Curie-Skłodowska University \\ Pl. M. Curie-Skłodowskiej 1, 20-031 Lublin, Poland
}

(Received August 17, 1999)

\begin{abstract}
We study the charge exchange in atom-metal collisions in the framework of the generalized time-dependent Anderson-Newns model. The electron correlations and correlated hopping are treated within the mean-field approximation. The resulting one-particle model with an effective spin-dependent atom-surface coupling is used to study the charge transfer in scattering of $\mathrm{Na}$ and $\mathrm{Li}$ atoms on metal surfaces. It is shown that the effective occupation dependent atom-surface coupling influences mainly the expectation values for producing positive, neutral, and negative particles for small work functions and high atom velocities. It is also shown that the temperature dependence of these expectation values is more visible, especially for magnetic solutions.
\end{abstract}

PACS numbers: 79.20.Rf, 78.70.-g, 82.65.Pa

\section{Introduction}

Electronic charge transfer between atoms or ions and metal surfaces has attracted much attention in recent years (e.g. [1-3]). Understanding of this typically nonadiabatic phenomenon is of primary importance in many surface analysis techniques like secondary-ion mass spectroscopy or ion-scattering spectroscopy. In addition, the most advanced methods to generate intense negative hydrogen beams used for the heating of fusion plasma make use of the negative surface ionization. The main characteristics of such scattering experiment are the outgoing-atom charge state probabilities or electron occupation numbers of the atom orbital. The most widely used theoretical model to describe the charge exchange process and to calculate the final charge states of the outgoing atoms is based on the Anderson-Newns (AN) Hamiltonian [4]. This model was successfully used in the chemisorption theory and its time-dependent version is very well suited to describe the dynamical processes. Usually, the centre of mass of the atom is assumed to move along a classical trajectory while the $z$-dependent parameters ( $z$ being the distance between an atom and metal surface) of the AN Hamiltonian parameters can be easily converted into the time-dependent ones. In the first theoretical treatments of the charge transfer, the Coulomb electron-electron interaction was neglected. As a result, the required solution of the problem was obtained in a closed form (e.g. [4-6]). However, if one calculates the different final charge state 
probabilities of the outgoing atom then all possible ionization channels for the atom have to be taken into account. In many theoretical calculations of the charge transfer within the time-dependent AN (TDAN) model, with the included electron correlation effects, the Hartree-Fock (HF) approximation was used. For example, in Ref. [7] the positive and negative ionization probability of an atom reflected from a metal surface was calculated using the time-dependent HF approximation. The exact evolution operator was replaced by the approximate time evolution operator obeying the equation of motion with HF Hamiltonian. In Ref. [8] the intra-atomic Coulomb correlation was taken into account as an infinity quantity and thus the double occupation of an atomic orbital was not allowed. In Ref. [9] the HF one-particle model was used to calculate the probabilities of the different charge states of an atom scattered by semi-infinite linear chains. Yoshimori et al. [10] (see also [3]) used the equation of motion method within HF approximation and showed that the resulting one-particle model is characterised by the effective atom ionization level

$$
\varepsilon_{A}^{\sigma}(t)=\varepsilon_{A}(t)+U(t)\left\langle n_{A-\sigma}(t)\right\rangle .
$$

Here, $\varepsilon_{A}(t)$ and $U(t)$ represent the time-dependent atom ionization level and on-atom Coulomb electron-electron repulsion, respectively. Now, the atom orbital occupancies can be obtained following, for example, Brako and Newns [4] with one significant difference: the system of two integral equations for $\left\langle n_{A \sigma}(t)\right\rangle$ and $\left\langle n_{A-\sigma}(t)\right\rangle$ should be solved simultaneously instead of a given one simple finite expression for $\left\langle n_{A}(t)\right\rangle$, being the solution of the spinless model.

In all papers cited above, the authors assumed that the electron hopping matrix elements $V_{A k}(t)$ are independent of the orbital occupation numbers of the scattered atoms. However, this assumption may be incorrect, as was mentioned by Hellsing and Zdanov [11]. The idea of the occupation number dependent hopping matrix elements comes from the general quantum mechanical description of an electron tunnelling in condensed matter. It has been shown that the probability of an electron tunnelling from a donor to an acceptor (lying at a distance $R$ one from another) in condensed matter can be roughly written as proportional to $\exp (-2 R \gamma)$, where $\gamma$ can be expressed in terms of the donor ionization energy $\varepsilon_{d}, \gamma^{2}=2 m \varepsilon_{d} / \hbar^{2}$ [12]. On the other hand, it is known that if the charge transfer between the metal surface and an atom scattered on it is described by means of the TDAN model treated in the HF approximation, then the resulting one-particle model is characterised by the effective atom ionization level given by Eq. (1). Then, from the electron tunnelling theory in a condensed matter one can imply $[11,12]$ that

$$
V_{A k}^{2}(z)=V_{A k}^{2}(0) \exp \left(-2 z \gamma_{\sigma}\right)
$$

where $\gamma_{\sigma}^{2}=2\left|\varepsilon_{A}(z)+U(z)\left\langle n_{A-\sigma}(z)\right\rangle\right|$ (in atomic units). One should remember, however, that such comparison of different electron tunnelling processes is of limited applicability only. For example, the electron transfer from a donor to an acceptor is a transition between discrete states while in the case of atoms scattered on metal surfaces, the electron transitions are between discrete and extended states. 
In this paper we explore another form of the effective spin dependent hopping matrix elements. This occupation dependence of $V_{A k}(z)$ appears in a natural way in the generalised AN model [13]. It is known that the adatom electron charge, calculated for example for hydrogen chemisorbed on the transition metal surfaces, has too high value compared to experimental data $[14,15]$. Recently, we have analysed the chemisorption phenomenon using the AN Hamiltonian supplemented with, the so-called, correlated hopping $(\mathrm{CH})$ terms. These terms correspond to the transition of the substrate electron with spin $\sigma$ to an atomic orbital when the second atomic orbital with spin $-\sigma$ is already occupied. The chemisorption calculations performed for such generalized AN model give much more realistic picture of the hydrogen adsorption on transition metal surfaces [16].

Recently, we have investigated the charge transfer in atom-metal surface collisions and calculated $\left\langle n_{A \sigma}(t)\right\rangle$ for model in which the $\mathrm{CH}$ terms were incorporated [17]. It appeared that for constant values of $\varepsilon_{A}$ and $U$ the influence of the $\mathrm{CH}$ on $\left\langle n_{A \sigma}(t)\right\rangle$ depends on the relative position of the ionization and affinity levels as well as on the Fermi level. For paramagnetic solution, the $\mathrm{CH}$ increases $\left\langle n_{A \sigma}(t)\right\rangle$ as the affinity level is moving down $\left(\varepsilon_{A}<E_{\mathrm{F}}, \varepsilon_{A}+U / 2<E_{\mathrm{F}}\right)$. The largest influence is observed for $\varepsilon_{A}$ and $\varepsilon_{A}+U$ lying below the Fermi energy. For $\varepsilon_{A}\left\langle\varepsilon_{\mathrm{F}}\right.$ and $\varepsilon_{A}+U / 2>E_{\mathrm{F}},\left\langle n_{A \sigma}(t)\right\rangle$ decreases with $\varepsilon_{A}$ moving up and the largest differences, in comparison with the TDAN model without CH is observed for both $\varepsilon_{A}$ and $\varepsilon_{A}+U$ lying above the Fermi level. The occupancies of different charge states respond to the $\mathrm{CH}$ in a more sophisticated manner. For example, for the paramagnetic solutions and $\varepsilon_{A}\left\langle E_{\mathrm{F}}, \varepsilon_{A}+U\right\rangle E_{\mathrm{F}}$, the fraction of the negative ions is enhanced while other fractions (neutrals and positive ions) are almost unchanged. These conclusions can be substantially changed for realistic $z$-dependent ionization and affinity levels and realistic atom resonance levels width. Depending on both the surface electronic characteristics and a kind of the scattered atoms, the atom ionization and affinity levels can be remarkably moved (relative to the Fermi energy) during the atom motion along its trajectory. Therefore, in order to give a quantitative estimation of the influence of $\mathrm{CH}$ on the charge transfer, the realistic $z$-dependent atom resonance level width as well as $z$-dependent atom ionization and affinity levels should be included into consideration. In this paper the results of such calculations are reported. In the second section the model Hamiltonian and the main assumptions of the used approximations are presented. The third section contains the obtained results, discussion, and conclusions.

\section{Model}

We describe the system of the moving atom and metal surface by the time-dependent AN Hamiltonian augmented by the correlated hopping terms

$$
\begin{aligned}
H= & \sum_{k \sigma} \varepsilon_{k} n_{k \sigma}+\sum_{\sigma} \varepsilon_{A}(t) n_{A \sigma}+U(t) n_{A \uparrow} n_{A \downarrow}+\sum_{k \sigma}\left[V_{A k}(t) a_{A \sigma}^{+} a_{k \sigma}+\text { h.c. }\right] \\
& -\sum_{k \sigma}\left[V_{A A A k}(t) n_{A-\sigma} a_{A \sigma}^{+} a_{k \sigma}+\text { h.c. }\right]
\end{aligned}
$$

where $\varepsilon_{A}(t)$ and $\varepsilon_{k}$ are the energy level of an orbital of the moving atom and energy level of the metal electron states, respectively; $U(t)$ is the intra-atomic Coulomb in- 
teraction between electrons with opposite spins and $V_{A k}(t)$ represents the coupling between the moving atom and the metal surface. The operator $a_{A \sigma}^{+}\left(a_{A \sigma}\right)$ creates (annihilates) a spin $\sigma$ electron in the atomic orbital, $n_{A \sigma}=a_{A \sigma}^{+} a_{A \sigma}, a_{k \sigma}^{+}\left(a_{k \sigma}\right)$ creates (annihilates) a spin $\sigma$ electron in the metallic state and $n_{k \sigma}=a_{k \sigma}^{+} a_{k \sigma}$. The matrix elements $V_{A A A k}(t)$ represent a time-dependent version of $V_{A A A k}$ defined as follows [13]:

$$
\dot{V}_{A A A k}=\iint \mathrm{d} r_{1} \mathrm{~d} r_{2} \varphi_{A}^{*}\left(r_{1}\right) \varphi_{A}^{*}\left(r_{2}\right) \frac{e^{2}}{\left|r_{1}-r_{2}\right|} \varphi_{A}\left(r_{2}\right) \varphi_{k}\left(r_{1}\right),
$$

where $\varphi_{A}(r)$ and $\varphi_{k}(r)$ are wave functions of the atomic and metal states, respectively. We approximate the matrix elements $V_{A A A k}(t)$ as $V_{A A A k}(t)=\xi V_{A k}(t)$, $0 \leq \xi \leq 1$. We have used this approximation in the chemisorption calculations and found that it works relatively well [18]. We suppose a scattered atom moves along the classical trajectory, so the time-dependence of model parameters is considered to be given along the trajectory.

In the following we calculate the electron occupation number of a moving atom, $\left\langle n_{A \sigma}(t)\right\rangle$, where $\left\langle n_{A \sigma}(t)\right\rangle=\left(\psi(t)\left|n_{A \sigma}\right| \psi(t)\right)$ and $\left.\mid \psi(t)\right)$ satisfies the time-dependent Schrödinger equation. We calculate also the expectation values for producing positive, neutral, and negative scattered particle (in the HF approximation):

$$
\begin{aligned}
& I^{+}(t)=\left(1-\left\langle n_{A \uparrow}(t)\right\rangle\right)\left(1-\left\langle n_{A \downarrow}(t)\right\rangle\right), \\
& I_{\sigma}^{0}(t)=\left\langle n_{A \sigma}(t)\right\rangle\left(1-\left\langle n_{A-\sigma}(t)\right\rangle\right), \\
& I^{-}(t)=\left\langle n_{A \sigma}(t)\right\rangle\left\langle n_{A \sigma}(t)\right\rangle .
\end{aligned}
$$

The mean-field treatment of the model defined in Eq. (3) with constant parameters was described in [17]. For the sake of completeness we shall briefly outline here the main points of the derivation of the final differential equations for $\left\langle n_{A \sigma}(t)\right\rangle$. Starting with the equation of motion for $\left\langle n_{A \sigma}(t)\right\rangle$, one obtains the equation in which the function $\left\langle a_{A \sigma}^{+}(t) a_{k \sigma}(t)\left(1-\xi n_{A-\sigma}(t)\right)\right\rangle$ appears. We decouple it as follows (cf. [19]):

$$
\left\langle a_{A \sigma}^{+}(t) a_{k \sigma}(t)\left(1-\xi n_{A-\sigma}(t)\right)\right\rangle \simeq\left(1-\xi\left\langle n_{A-\sigma}(t)\right\rangle\right)\left\langle a_{A \sigma}^{+}(t) a_{k \sigma}(t)\right\rangle .
$$

Using this decoupling and the corresponding solution for $a_{k \sigma}(t)$ one can obtain the equation for $\mathrm{d}\left\langle n_{A \sigma}(t)\right\rangle / \mathrm{d} t$ with additional unknown function $\left\langle a_{k \sigma}^{+}\left(t_{0}\right) a_{A \sigma}(t)\right\rangle$. Writing down the equation of motion for this function and taking into consideration only the most important term in this equation, $\left\langle n_{A-\sigma}(t) a_{q \sigma}^{+}(t) a_{k \sigma}\left(t_{0}\right)\right\rangle$, decoupled as indicated in Eq. (6), we obtain the following set of two equations (for spin-up and spin-down):

$$
\begin{aligned}
& \frac{\mathrm{d}}{\mathrm{d} t}\left\langle n_{A \sigma}(t)\right\rangle=\Delta_{\sigma}(t)\left(1-2\left\langle n_{A \sigma}(t)\right\rangle\right)-2 k T \Delta_{\sigma}^{1 / 2}(t) \int_{t^{\prime}}^{t} \mathrm{~d} t^{\prime} \Delta_{\sigma}^{1 / 2}\left(t^{\prime}\right) \\
& \quad \times \sin \left[\int_{t^{\prime}}^{t} \mathrm{~d} t^{\prime \prime}\left(\varepsilon_{A}\left(t^{\prime \prime}\right)+U\left(t^{\prime \prime}\right)\left\langle n_{A-\sigma}\left(t^{\prime \prime}\right)\right\rangle\right)\right] \exp \left[-\int_{t^{\prime}}^{t} \mathrm{~d} t^{\prime \prime} \Delta_{\sigma}\left(t^{\prime \prime}\right)\right] \\
& \quad / \sinh \pi k T\left(t-t^{\prime}\right)
\end{aligned}
$$

where $\Delta_{\sigma}(t)=\pi \sum_{k}\left|V_{A k}\right|^{2}\left(1-\xi\left\langle n_{A-\sigma}(t)\right\rangle\right)^{2} \delta\left(E-\varepsilon_{k}\right)|u(t)|^{2}$ and $T$ is a temperature. 
Here it was assumed that the hopping matrix elements are separable, i.e. $V_{A k}(t)=V_{A k} u(t)$ and that the density of metal surface electron states multiplied by the square of $V_{A k}$ is a constant function. Now it is clear that the mean field treatment of the correlated hopping results in atom orbital occupation dependence of the effective hopping matrix elements. More elaborate mean-field calculations do not change the form of the final expression (7). The only difference is the replacement $\varepsilon_{A}+U\left\langle n_{A-\sigma}\right\rangle \rightarrow \varepsilon_{A}+U\left\langle n_{A-\sigma}\right\rangle+\xi R^{-\sigma}$, where $R^{-\sigma}(t)$ is a complicated function of time [17]. The calculations of $\left\langle n_{A \sigma}(t)\right\rangle$ done for constant values of $\varepsilon_{A}, U$, and narrow surface energy bands show that in a long-time limit this additional renormalization of $\varepsilon_{A}$ does not introduce any qualitative changes in comparison with results obtained within simpler approach, Eq. (7). Therefore, having this in mind, in the following we give the analysis of $\left\langle n_{A \sigma}(t)\right\rangle$ obtained on the basis of Eq. (7).

Input to the calculations of the atom orbital occupancy $\left\langle n_{A \sigma}(t)\right\rangle$ or the charge state fractions $I_{\sigma}^{0}(t), I^{-}(t), I^{+}(t)$ are the energies and width of the atomic level. As an atom approaches the surface, the atom ionization and affinity levels are shifted by the image potential and broadened into a resonance of width $\Delta(z)$, $z$ being the distance of the atom from the surface. The most popular forms of $\Delta(z)$ correspond to exponential or Gaussian dependence on the distance $z$ (see, however [20]). In our calculations we have used a Gaussian form for $\Delta(z)$ :

$$
\Delta \rightarrow \Delta(z)=\Delta_{0} \exp \left(-\gamma z^{2}\right),
$$

with appropriately chosen values for $\Delta_{0}$ and $\gamma$ [21].

We allow for $\varepsilon_{A}(z)$ and $U(z)$ to vary along the trajectory from the values corresponding to the free atom case, to the values which are appropriate for the atom localized near the surface. We use, for $\varepsilon_{A}$ and $U$, two forms of the $z$-dependence. The first, very often used in the literature, e.g. [11, 22], takes into account the image interaction in its canonical form which in atomic units reads

$$
\begin{aligned}
& \varepsilon_{A}(z)=\varepsilon_{A}(\infty)+\varphi+\frac{1}{4\left(z+z_{0}\right)}, \\
& U(z)=-\varepsilon_{A}(\infty)+A(\infty)-\frac{1}{2\left(z+z_{0}\right)},
\end{aligned}
$$

where $\varepsilon_{A}(\infty)(A(\infty))$ is the ionization (affinity) energy level, $\varphi$ is the work function and $z_{0}$ is the parameter which takes into account the image shift saturation when the atom gets close to the surface. As the second form for $z$-dependence of the ionization and affinity levels, we take the expressions given in Ref. [23], where the image-like behaviour at a distance close to the surface is more carefully described using the following Gaussian fit:

$$
\begin{aligned}
& \varepsilon_{A}(z)=a_{1} \exp \left(-\alpha z^{2}\right)+a_{2}, \\
& \varepsilon_{A}(z)+U(z)=a_{3} \exp \left(-\alpha z^{2}\right)+a_{4},
\end{aligned}
$$

where $a_{1}, a_{2}, a_{3}, a_{4}$, and $\alpha$ depend on both the characteristics of the scattered atom and the surface work function. 


\section{Numerical results, discussion, and conclusions}

In this section we present the results obtained for occupation numbers $\left\langle n_{A \sigma}(z)\right\rangle$ as a function of atom-surface distance $z$ as well as for probabilities of formation of the charged and neutral atom states versus the work function. The comparison with the results obtained within the standard TDAN model is also given. In order to demonstrate the consequences of the occupation number dependence on the hopping matrix elements we performed the calculations for small and large velocities of the scattered atoms and for different parametrization scheme of the $z$-dependence of $\varepsilon_{A}(z)$ and $U(z)$. We have also analyzed the temperature dependence of $\left\langle n_{A \sigma}(t)\right\rangle$ and the memory effects.

The time dependence of the model parameters $\varepsilon_{A}, U$, and $V_{A k}$ enters via the ion trajectory. We use the classical trajectory approximation and take

$$
z(t)= \begin{cases}s_{0}-v t & \text { for } t<s_{0} / v \\ v t-s_{0} & \text { for } t \geq s_{0} / v\end{cases}
$$

where $s_{0}$ denotes the initial point from which the atom starts to move towards the surface (time $t=0$ ) and $v$ is the perpendicular (to the surface) component of the atom velocity (here we ignore parallel velocity).

In our calculations the atom velocity $v$ is taken to be 0.01 or 0.1 (in atomic units). These values of $v$ correspond to conditions usually met in experiments. For example, in experiments with lithium atoms these velocities correspond to kinetic energies extending from about $17 \mathrm{eV}$ to $1700 \mathrm{eV}$.

In order to investigate the dynamics of the charge transfer one should consider the motion of the atom from infinitely large distances towards the surface and then, after the scattering on the surface, again to infinitely large distances. However, as the matrix hopping elements are strongly decaying functions of the distance $z$, then it is sufficient to start from finite value of $z$. In our calculations we took $s_{0}$ to be 20 a.u. After scattering on the surface, the atom motion along outward way should be considered until the solutions for $\left\langle n_{A \sigma}(z)\right\rangle$ or $I^{+}(z), I^{-}(z)$, and $I_{\sigma}^{0}(z)$ will be stabilized. Although the asymptotic (for $z=\infty$ ) values of the probabilities of being charged or occupation numbers for the moving particle can be compared with the experimental data, we give also the time evolution on the whole atom trajectory in order to clarify the effects of the correlated hopping and initial conditions on the charge transfer process.

In Figs. 1-6 we present results corresponding to the $\mathrm{Na}$ atoms scattered on surfaces with different work functions. The atomic resonance width $\Delta(z)$ is taken in the Gaussian form with $\Delta_{0}=5 \mathrm{eV}, \gamma=0.05 \mathrm{bohr}^{-2}$ and the image-potential-modified affinity and ionization levels are assumed, Eq. (9) [11, 21]. For the parameter $z_{0}$ which reduces the image interaction near the metal surface we take the value 3 a.u. [11].

In Figs. 1 and 2 we give the evolution of the occupancies $\left\langle n_{A \sigma}\right\rangle$ along the way towards the surface (negative values of $z$ ) and on the outgoing way (positive values of $z$ ). The upper parts (a) and (b) correspond to the paramagnetic initial conditions, i.e. $\left\langle n_{A \downarrow}(z=-\infty)\right\rangle=\left\langle n_{A \uparrow}(z=-\infty)\right\rangle=0$. The lower parts (c) and (d) represent results for magnetic initial conditions, i.e. $\left\langle n_{A \downarrow}(z=-\infty)\right\rangle=0$, $\left\langle n_{A \uparrow}(z=-\infty)\right\rangle=1$. The first case can be realized in experiments taking the 


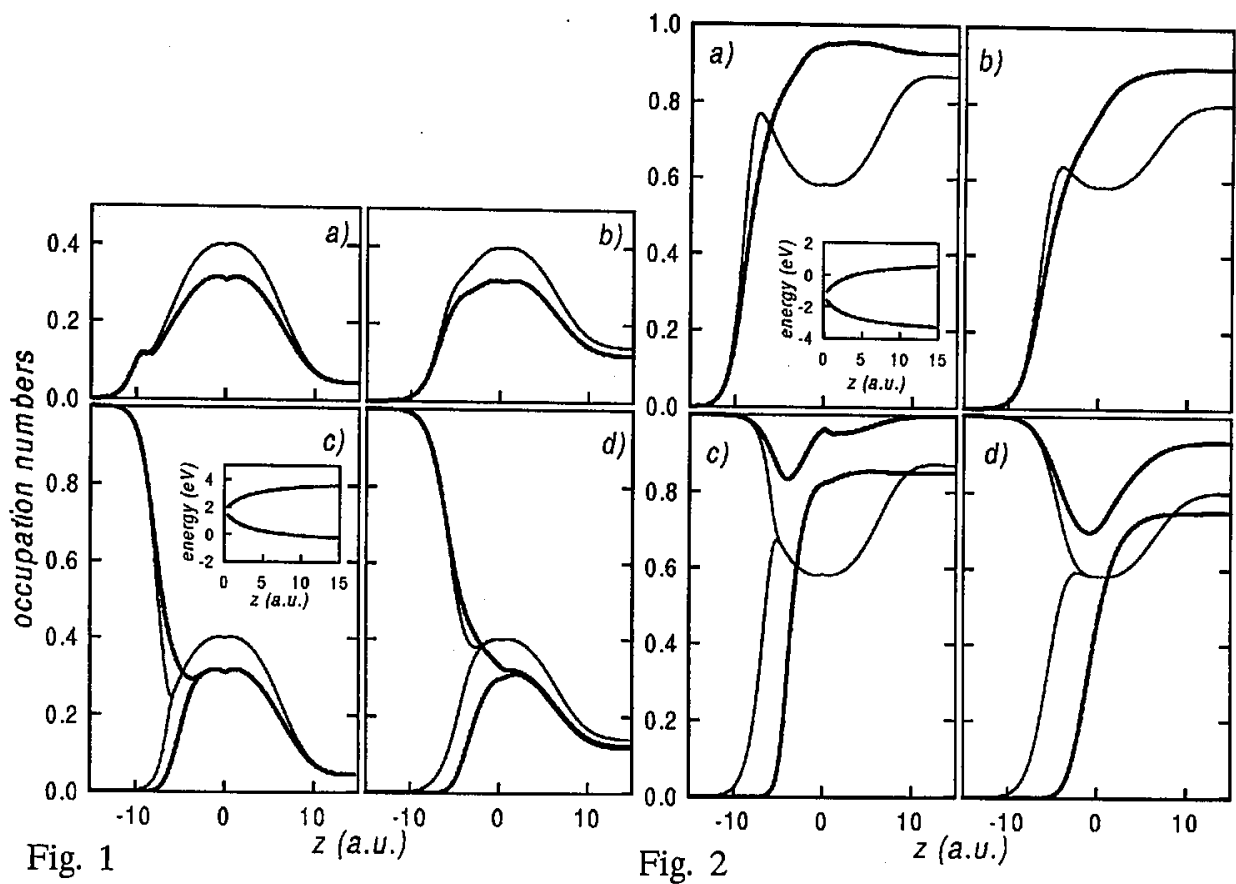

Fig. 1. Occupation numbers $\left\langle n_{A \sigma}\right\rangle$ of $\mathrm{Na}$ atom vs. distance $z$ from the surface for atom velocity $v=0.01$ a.u. ((a) and (c)) and for $v=0.1$ a.u. ((b) and (d)). Parts (a) and (b) correspond to non-magnetic initial conditions and parts (c) and (d) to magnetic ones. The thin (thick) lines correspond to $\xi=0(\xi=1)$. The atomic resonance width and affinity and ionization levels are calculated according to Eqs. (8), (9) with $\Delta_{0}=5 \mathrm{eV}$, $\gamma=0.05 \mathrm{bohr}^{-2}, \varepsilon_{A}(\infty)=5.14 \mathrm{eV}, A(\infty)=0.55 \mathrm{eV}$ and the work function $\varphi=4.5 \mathrm{eV}$. The negative values of $z$ correspond to the atom moving towards the surface and the surface temperature $T=300 \mathrm{~K}$. The insert in part (c) shows the energy dependent atom level.

Fig. 2. The same as in Fig. 1 but for the work function $\varphi=1.5 \mathrm{eV}$.

incoming atom beam of the positively ionized $\mathrm{Na}$ atoms. The second one can be realized by preparing the initial atom beam of neutral $\mathrm{Na}$ atoms with spin-up electron on the outer atomic shell. The surface work function $\varphi=4.5 \mathrm{eV}$ (Fig. 1) and $\varphi=1.5 \mathrm{eV}$ (Fig. 2) and the surface temperature $T=300 \mathrm{~K}$. In experiments, the work function can be changed by using different metal targets or taking one metal target, i.e. tungsten target partially covered by different amount of alkali atoms. The thin lines correspond to the results obtained for standard TDAN model and thick lines for model given by Eq. (3) with the $\mathrm{CH}$ included and $\xi=1$.

We observe that for large work function (Fig. 1) at small atom velocity the asymptotic values of the occupation numbers do not depend on the $\mathrm{CH}$, both for magnetic and non-magnetic initial conditions. However, the $\mathrm{CH}$ influences the charge transfer for small atom-surface distance. For higher atom velocity, Fig. 1b,d, the influence of the $\mathrm{CH}$ is, in principle, similar as for smaller velocity, an important 
difference appears, however, in the asymptotic values of $\left\langle n_{A \sigma}\right\rangle$, which depend now on $\xi$. The $\mathrm{CH}$ decreases the occupation numbers almost on the whole trajectory from $z=-\infty$ to the surface and back to $z=+\infty$ for non-magnetic, as well as, for magnetic initial conditions.

We observe quite different situation for small work function (Fig. 2). Now, for small atom-surface separation both the ionization and affinity levels are below the Fermi level and the charge transfer between atom and metal surface can be more efficient. This can be most clearly observed for small atom velocity (compare Fig. 1a and Fig. 2a). For non-magnetic solutions the $\mathrm{CH}$ increases the occupation numbers on the whole atom trajectory (see Fig. 1a,b). For magnetic initial conditions, the situation is more complicated. First of all, the model with the $\mathrm{CH}$ included leads to the appearance of the memory effects. The phenomenon of the memory about the initial atom charge state can be roughly explained as follows. As the atom comes close to the surface, the electrons initially localized on atom and metal surface start to hop back and forth between atom and surface. If the atom spends some time long enough near the surface (for example, at small atom velocity) and electrons have a time to hop between atom and surface several times, then the memory about the initial atom change state can be to some extent erased [23].

The memory effect can be seen by comparing curves corresponding to $\xi=1$ with those corresponding to the standard TDAN model $(\xi=0)$, Fig. 2a,c for $v=0.01$ a.u. and Fig. $2 \mathrm{~b}, \mathrm{~d}$ for $v=0.1$ a.u. For small as well as for large atom velocities the memory effects are absent for model without $\mathrm{CH}$ (compare $n_{A \sigma}(\infty)$ (thin curves) on parts (a), (c), and (b), (d), respectively). However, for $\xi=1$, starting with magnetic initial values $\left\langle n_{\uparrow}(-\infty)\right\rangle=1,\left\langle n_{\downarrow}(-\infty)\right\rangle=0$, we obtain also magnetic solutions (Fig. 2c,d).

Figure 3 demonstrates how important is the localization of affinity and ionization levels close to the surface for small work functions, especially for small

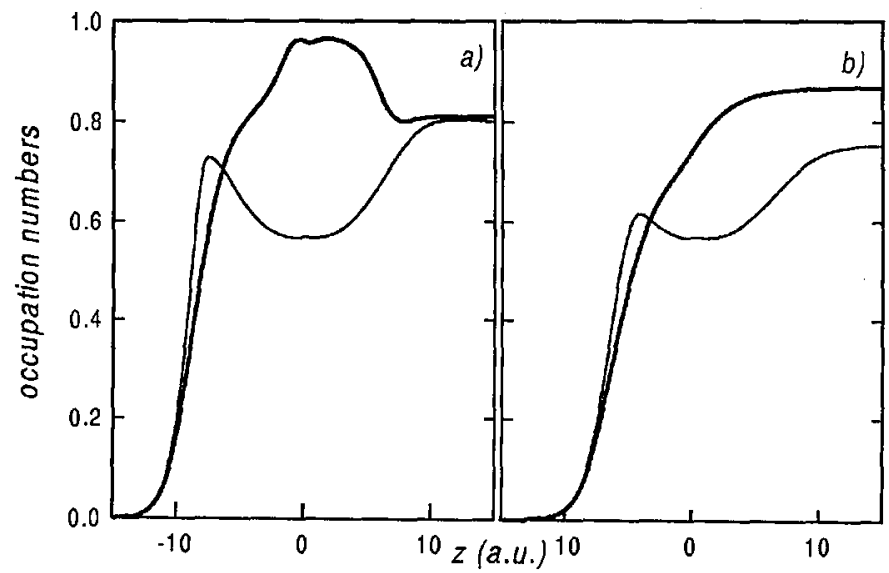

Fig. 3. The same as in Fig. 1 but for the work function $\varphi=1.75 \mathrm{eV}$ and for non-magnetic initial conditions, only. Part (a) (respectively (b)) corresponds to $v=0.01$ a.u. (0.1 a.u.). 


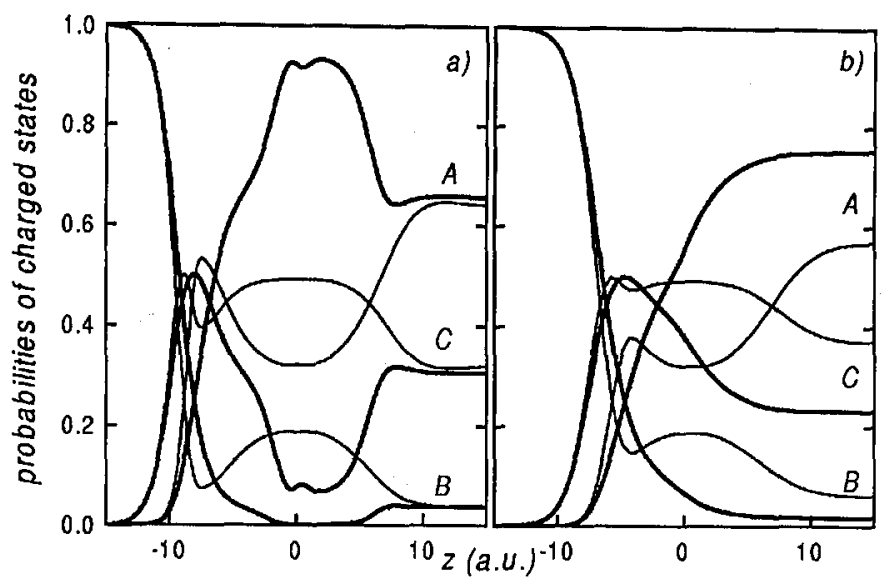

Fig. 4. Probabilities of the different charge states $I^{-}, I^{+}$, and $I^{0}=I_{\uparrow}^{0}+I_{\downarrow}^{0}$, curves $A, B$ and $C$, respectively, for parameters as in Fig. 3 .

atom velocities. Now the work function $\varphi=1.75 \mathrm{eV}$ and the occupation numbers for $\xi=1$ differ substantially from those corresponding to $\varphi=1.5$ (Fig. 2a). For higher atom velocity, the general behaviour of $\left\langle n_{A \sigma}(z)\right\rangle$ is similar for both these work functions and only the asymptotic values are slightly smaller for $\varphi=1.75 \mathrm{eV}$.

Figure 4 shows the $z$-dependence of the probabilities for formation of $\mathrm{Na}^{+}$, $\mathrm{Na}$, and $\mathrm{Na}^{-}$(curves $A, B$, and $C$, respectively) for the atom velocity $v=0.01$ a.u. (part (a)), $v=0.1$ a.u. (part (b)). The initial conditions and other parameters are the same as in Fig. 3a,b. One observes a rather small influence of the $\mathrm{CH}$ on asymptotic values of $I^{-}, I^{+}$, and $I^{0}$ for small atom velocity. On the other hand, for high atom velocity this influence is significant, especially for $I^{-}$and $I^{0}$.

Figure 5 presents the work function dependence of the probabilities for formation of $\mathrm{Na}^{+}, \mathrm{Na}$, and $\mathrm{Na}^{-}$(curves $A, B$, and $C$, respectively) for the velocities $v=0.01$ a.u. (left part) and $v=0.1$ a.u. (right part). The calculations were done for the case of an initially positive ionized $\mathrm{Na}$ atom for $\xi=0$ (thin curves) and for $\xi=1$ (thick curves). For small atom velocity, the $\mathrm{CH}$ influences slightly the final probabilities of being in different charge states only for small work functions. This influence is more pronounced for high atom velocity, especially for low work functions. Such behaviour can be explained as follows. The correlated hopping increases significantly the production of negative ions when the ionization and affinity lie below the Fermi level [17]. For small work function $\varphi=1.75 \mathrm{eV}$, the affinity level crosses the Fermi level at a distance $z \approx 2.5$ a.u. from the surface. The $z$-dependence of the curve $A$ (for $\xi=1$ ) in Fig. 4a shows that the largest enhancement of $I^{-}$is observed for that region of the particle trajectory for which both atomic levels are below the Fermi level. As the velocity is relatively low, then the system has longer time to equilibrate to lower values of $I^{-}$on this part of an atom trajectory for which affinity level is above the Fermi energy. For higher velocity, Fig. $4 \mathrm{~b}$, the time for system to equilibrate is shorter and $I^{-}(\infty)$ is greatly enhanced over $\xi=0$ curve in comparison with small velocity case, curve $A$ for $\xi=1$. 


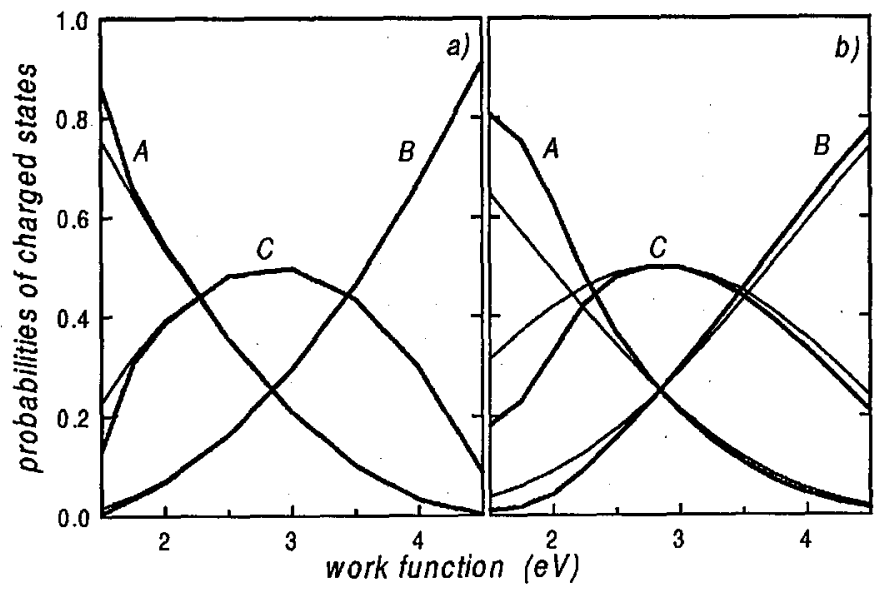

Fig. 5. Asymptotic values of the probabilities of different $\mathrm{Na}$ charge states $\mathrm{I}^{-}, \mathrm{I}^{+}$, and $I^{0}$, curves $A, B$, and $C$, respectively, vs. the work function. Parts (a) and (b) correspond to the atom velocity $v=0.01$ a.u. and 0.1 a.u., respectively. Other parameters as in Fig. 3.

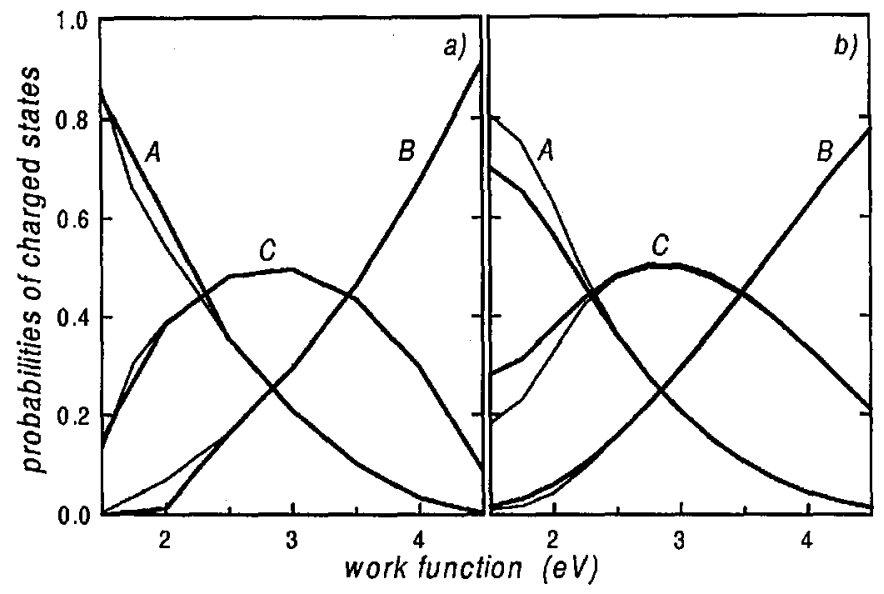

Fig. 6. Analysis of the memory effect for parameters as in Fig. 5 but thin (thick) lines are obtained for non-magnetic (magnetic) initial conditions. Parts (a) and (b) correspond to the atom velocity $v=0.01$ a.u. and 0.1 a.u., respectively.

Figure 6 displays a comparison between results obtained for non-magnetic (thin curves) and magnetic (thick curves) initial conditions for model with $\xi=1$ and for $v=0.01(v=0.1$ ) a.u., part (a) (respectively (b)). All other parameters are the same as in Fig. 5. Again, the main differences appear only for small work functions and, at the same time, these differences are greater for higher velocities. These results can be understand analyzing the occupation numbers curves given in Figs. 1 and 2. 


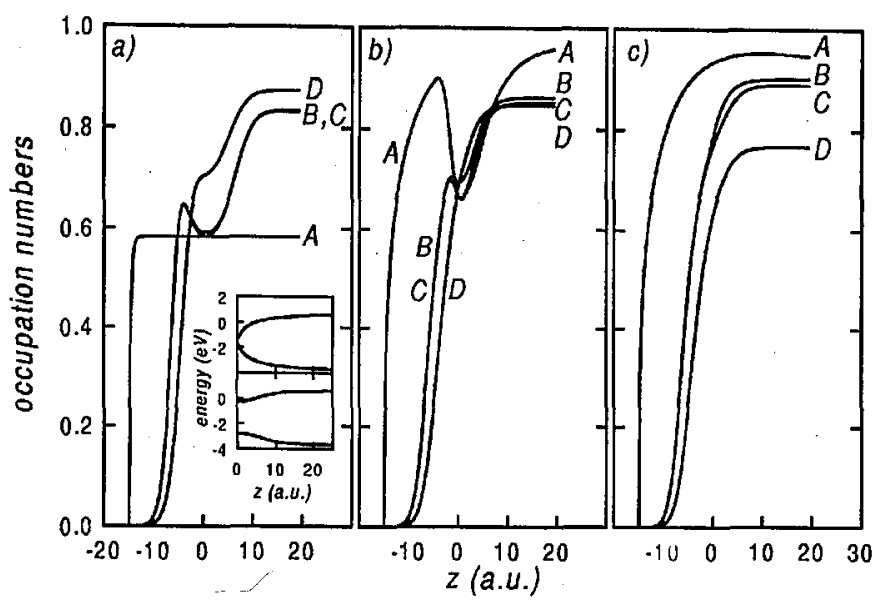

Fig. 7. Occupation numbers $\left\langle n_{A \sigma}\right\rangle$ of Li atom vs. the distance $z$ from the surface for atom velocity $v=0.1$ a.u., work function $\varphi=1.45 \mathrm{eV}$, and $T=300 \mathrm{~K}$ obtained for non-magnetic initial conditions. Parts (a), (c) correspond to $\xi=0$ (1) and part (b) to $z$-dependent $\xi$, Eq. (12). Curves $A, B, C$, and $D$ are calculated for different $z$-dependent $\varepsilon_{A}, U$, and $\Delta$ (see text). The insert in part (a) shows the energy-dependent atom levels according to Eq. (9) (upper part) and Eq. (10) (lower part).

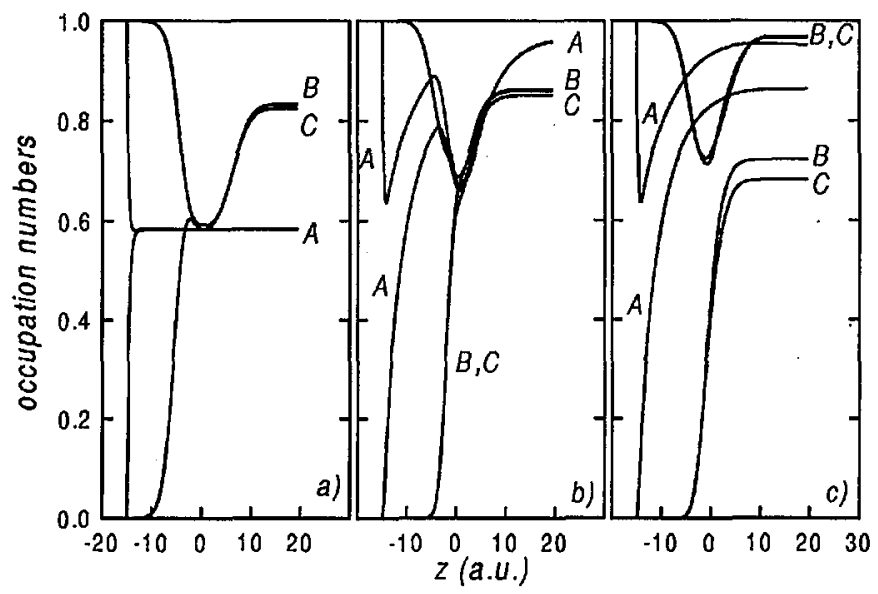

Fig. 8. The same as in Fig. 7 but for magnetic initial conditions.

Figures 7 and 8 show the $z$-dependence of the occupation numbers for lithium atoms with $v=0.1$ a.u. scattered on the metal surface with the work function $\varphi=1.45 \mathrm{eV}$ for non-magnetic and magnetic initial conditions, respectively. Parts (a) and (c) correspond to $\xi=0$ and $\xi=1$, respectively. Part (b) shows the results calculated for $z$-dependent $\mathrm{CH}$ strength $\xi$

$$
\xi(z)= \begin{cases}0.5+0.1 z & \text { for } z<5 \text { a.u. } \\ 1.0 & \text { for } z \geq 5 \text { a.u. }\end{cases}
$$

Our earlier estimation of the $\mathrm{CH}$ strength in the case of the hydrogen chemisorbed 
on the transition metal surface indicates that $\xi$ depends slightly on a distance between atom and the surface and takes its maximum value equal to 1.0 at a relatively small distance from the surface [18]. As the final charge state of the atom is determined mainly on the outgoing trajectory in some distance from the surface (see e.g. [22]), then one should expect that explicit $z$-dependence of the $\mathrm{CH}$ strength $\xi$ does not change remarkably $\left\langle n_{A \sigma}(\infty)\right\rangle$. On the other hand, at small distances the calculated curves can differ significantly. Our calculations support these remarks.

Curves $B, C$, and $D$ in Figs. 7 and 8 denote results for $\left\langle n_{A \sigma}(z)\right\rangle$ for different sets of the parametrization formulas. The curves $B$ are calculated according to the image-potential modified ionization and affinity levels, Eq. (9), with $\varepsilon_{A}(\infty)=$ $A(\infty), \varphi=1.45 \mathrm{eV}$ and $z_{0}=3$ a.u. (see the upper part of insert given in Fig. 7a). The curves $C$ are obtained for $\varepsilon_{A}(z)$ and $\varepsilon_{A}(z)+U(z)$ calculated according to the parametrization given in Ref. [21], Eq. (10) - the lower part of insert in Fig. 7a. In both cases we take $\Delta(z)$ with $\Delta_{0}=5 \mathrm{eV}$ and $\gamma=0.05 \mathrm{bohr}^{-2}$. Again, despite some differences for $z$-dependence of $\varepsilon_{A}$ and $U$ close to the surface, the asymptotic values of $\left\langle n_{A \sigma}(z)\right\rangle$ are very similar to each other. Such a behaviour is a consequence of the fact that at the distances where the final occupancies are formed both parametrizations give similar results for $\varepsilon_{A}$ and $\varepsilon_{A}+U$. However, an observable effect of the $\mathrm{CH}$ appears. With increasing $\xi$ the value of $\left\langle n_{A \sigma}(\infty)\right\rangle$ increases and also the difference between both parametrizations appears. Since in the literature various values of $\Delta_{0}$ are used, we have checked the influence of this parameter on the electron occupancy $\left\langle n_{A \sigma}(z)\right\rangle$ for different choices of the $\mathrm{CH}$ strength $\xi$. The results for $\Delta_{0}=1 \mathrm{eV}$ are shown as curves $D$ in Fig. 7. It appears that the influence of $\Delta_{0}$ is enhanced in combination with increasing $\mathrm{CH}$ strength $\xi$. In Figs. 7 and 8 we give also for comparison the results obtained for constant values $\varepsilon_{A}=-2.8 \mathrm{eV}, U=2.5 \mathrm{eV}$ and sudden switching-on the atom-surface interaction at $z=s_{0}$ (curves $A$ ). In this case both atomic levels lie below the Fermi level along the whole atom trajectory, so the $\mathrm{CH}$ strongly enhances the occupancy $\left\langle n_{A \sigma}(z)\right\rangle$ and the large differences are observable between the corresponding curves in parts (a), (b), and (c).

Figure 8 shows the case of the magnetic initial conditions. The differences between parametrizations (9) and (10) (curves $B$ and $C$ ) are more transparent than for non-magnetic initial conditions, especially for greater $\mathrm{CH}$ strength $\xi$. In addition, with increasing values of $\xi$, the final results for electron occupancies are more and more magnetic, i.e. $\left\langle n_{A \uparrow}(\infty)\right\rangle \neq\left\langle n_{A \downarrow}(\infty)\right\rangle$. Despite of the magnetic initial conditions, we observe non-magnetic asymptotic behaviour for $\xi=0$ with slight difference between $B$ and $C$ curves, part (a). For $z$-dependent values of $\xi$, part (b), the dynamics of the electron occupancy is more complicated although the asymptotic values are still non-magnetic.

If the $\mathrm{CH}$ strength takes the highest value $\xi=1$ on the whole atom trajectory, part (c), Fig. 8, then the qualitative differences occur. The solutions for $\left\langle n_{A \sigma}(z)\right\rangle$ appear to be magnetic ones and are more sensitive to various parametrization for $z$-dependence of the atomic levels.

Figure 9 shows the temperature dependence of the occupation number $\left\langle n_{A \sigma}(z)\right\rangle$ obtained for $\mathrm{Na}$ atoms, scattered on the metal surface of low work func- 


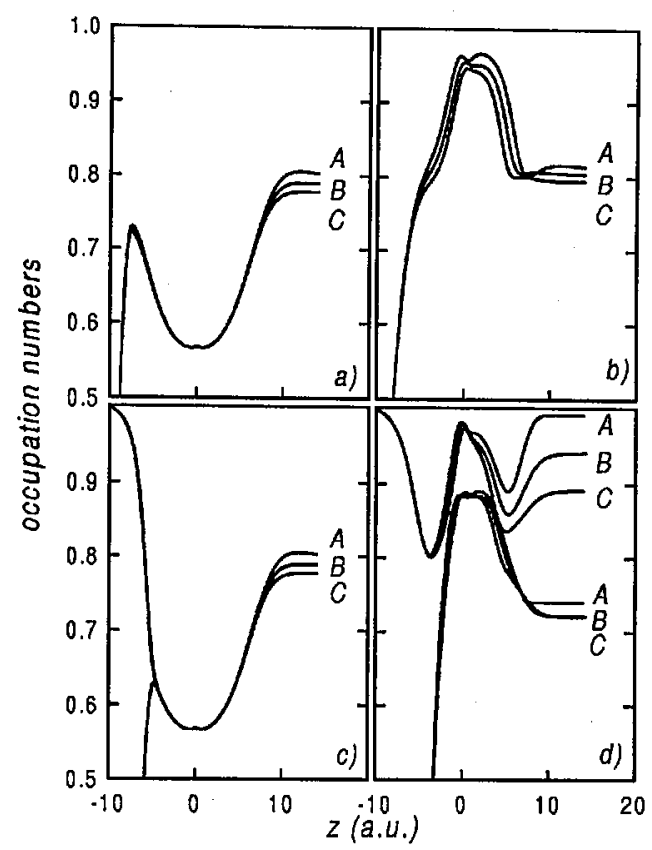

Fig. 9. The $z$-dependence of the occupation numbers for surface temperatures $T=$ 100,1000 , and $1500 \mathrm{~K}$, curves $A, B$ and $C$, respectively, for $\mathrm{Na}$ atoms moving with $v=0.01$ a.u. and scattered on surface with work function $\varphi=1.75 \mathrm{eV}$. Parts (a) and (b) ((c) and (d)) correspond to non-magnetic (magnetic) initial conditions and left (right) parts describe $\xi=0(\xi=1)$ case.

tion $\varphi=1.75 \mathrm{eV}$. The atom velocity $v=0.1$ a.u. and the initial conditions are taken in the form $\left\langle n_{A \sigma}(z=-\infty)\right\rangle=0$ for upper parts and $\left\langle n_{A \uparrow}(z=-\infty)\right\rangle=1$, $\left\langle n_{A \downarrow}(z=-\infty)\right\rangle=0$ for lower parts. Parts (a) and (c) correspond to the standard TDAN model $(\xi=0)$ and parts (b) and (d) to the model with the $\mathrm{CH}$ hopping included, $\xi=1$. Curves $A, B$, and $C$ are calculated for the surface temperature $T=100,1000$, and $1500 \mathrm{~K}$, respectively.

Let us discuss first the non-magnetic case (Fig. 9a,b). Since the work function is small, then both atomic levels lie below the Fermi level in the small distance from the surface. Therefore, the correlated hopping increases the occupation numbers, especially in the vicinity of the turning point at the surface (compare the curves on parts (a) and (b) and also on (c) and (d) at $z=0$ ).

The temperature effect is approximately the same for $\xi=0$ and $\xi=1$ - with increasing surface temperature the occupation numbers are smaller and smaller although the effect is not significant. The explanation of such behaviour is rather simple (see e.g. [21]). For non-zero temperatures the non-occupied states appear below the Fermi level in the metal. Then, the number of electrons which can hop between the metal and atom is reduced, especially when the atom is close to the surface. Electrons in the occupied states which appear above the Fermi level do not influence the charge state of the outgoing atom. These electrons can be transferred to the atom only if the atoms are in some distance from the surface 
(energy conservation) but in this case the atom-surface coupling is too small to influence considerably the atom occupation numbers.

In the case of the magnetic initial conditions, the influence of the $\mathrm{CH}$ is more complicated. First of all, for $\xi=0$ the asymptotic values of the occupation numbers are non-magnetic and for $\xi=1$ they are magnetic ones. The temperature behaviour for $\xi=0$ is similar to the one shown in Fig. 9a. For $\xi=1$, the temperature strongly influences only $\left\langle n_{A \uparrow}(z)\right\rangle$ and the changes of $\left\langle n_{A \downarrow}(z)\right\rangle$ are clearly smaller. Essentially, the same arguments as in the case of the non-magnetic initial conditions can be given to explain the asymptotic values of $I^{+}, I^{-}$, and $I^{0}$. Generally, the temperature effect for $\xi=1$ is more distinct than for $\xi=0$. The atom probability of being neutral and positively ionized increases with increasing temperature and that of being negative ionized decreases.

We have also performed calculations for higher atom velocity $v=0.1$ a.u. and for higher work function $\varphi=4.5 \mathrm{eV}$. For small work function and high atom velocity the temperature and $\xi$ influence slightly the asymptotic values of the electron occupancy. In the case of higher work function, the $\mathrm{CH}$ does not change $\left\langle n_{A \sigma}(z)\right\rangle$ for any surface temperature. There is, however, one essential difference from the low work function case. Now, the asymptotic values of occupation numbers increase with the surface temperature. This fact can be easily explained since for higher work function both atomic levels lie above the Fermi level for atom being at the small distance from the surface.

In conclusion, we have studied the resonant charge transfer between the atom and metal surfaces. The time-dependent Anderson-Newns model with additional many-body terms named as correlated hopping was used. The Coulomb correlations and correlated hopping was treated in the mean-field approximation reducing the initial many-body problem to one-particle one with the renormalized atom-surface coupling. This renormalization leads to the electron occupation dependent (spin-dependent) hybridization matrix elements $V_{\boldsymbol{A} k}^{\sigma}(t)$.

We have investigated the influence of the correlated hopping on the electron occupancies of the atomic levels for $\mathrm{Na}$ and $\mathrm{Li}$ atoms scattered on metal surfaces. The $z$-dependent ionization and affinity levels and the metal-surface coupling was used. The calculations were done for low and high atom velocities as well as for different initial conditions. The combined effect of the correlated hopping and the surface temperature on the electron occupancies was also considered.

Our main results can be summarized as follows:

- the CH does not influence the asymptotic values of the occupation numbers $\left\langle n_{A \sigma}(\infty)\right\rangle$ for large work functions and small atom velocities, both for magnetic and non-magnetic initial condition. For higher atom velocities the occupation numbers are reduced on the whole atom trajectory, especially at small distances from the metal surface.

- for non-magnetic initial conditions and small work functions, the $\mathrm{CH}$ increases the occupation numbers for low as well as high atom velocities. For magnetic initial conditions, the situation is more complicated. Now, for $\xi=0$ the final solutions appear to be non-magnetic but for $\xi=1$ they are magnetic ones. In this case one of these solutions, $\left\langle n_{A \uparrow}(\infty)\right\rangle$, is considerably larger than that 
obtained for $\xi=0$ but the second one, $\left\langle n_{A \downarrow}(\infty)\right\rangle$, is lowered only slightly below the non-magnetic solutions at $\xi=0$.

- the $\mathrm{CH}$ influences slightly the probabilities for formation of $\mathrm{Na}^{+}, \mathrm{Na}$, and $\mathrm{Na}^{-}$at small atom velocities and small work functions but this influence is much stronger for higher atom velocities.

\section{References}

[1] R. Brako, D.M. Newns, Rep. Prog. Phys. 53, 655 (1989).

[2] J.P. Gauyacq, A.G. Borisov, J. Phys., Condens. Matter 10, 6585 (1998).

[3] A. Yoshimori, K. Makoshi, Prog. Surf. Sci. 21, 251 (1986).

[4] R. Brako, D.M. Newns, Surf. Sci. 108, 253 (1981).

[5] M. Tsukada, Prog. Theor. Phys. Suppl. 106, 257 (1991).

[6] S. Tsuneyuki, N. Shima, M. Tsukada, Surf. Sci. 186, 26 (1987).

[7] T.B. Grimley, V.C.J. Bhasu, K.L. Sebastian, Surf. Sci. 124, 305 (1983).

[8] R. Brako, D.M. Newns, Solid State Commun. 55, 633 (1985).

[9] K.L. Sebastian, V.C.J. Bhasu, T.B. Grimley, Surf. Sci. 110, L571 (1981).

[10] A. Yoshimori, H. Kawai, K. Makoshi, Prog. Theor. Phys. Suppl. 80, 203 (1984).

[11] B. Hellsing, V.D. Zhdanov, Surf. Sci. 274, 411 (1992).

[12] R.F. Khairutdinov, K.I. Zamaraev, V.P. Zhdanov, in: Electron Tunneling in Chemistry, Comprehensive Chemical Kinetics, Vol. 30, Ed. R.G. Compton, Elsevier, Amsterdam 1989.

[13] E. Taranko, R. Taranko, Int. J. Mod. Phys. B 8, 1893 (1994).

[14] D.M. Newns, Phys. Rev. 178, 1123 (1968).

[15] K. Christmann, Surf. Sci. Rep. 9, 1 (1988).

[16] V.K. Fedyanin, G.M. Gavrilenko, R. Taranko, E. Taranko, Surf. Sci. 230, 329 (1990).

[17] R. Taranko, E. Taranko, Surf. Sci. 441, 167 (1999).

[18] E. Taranko, G.M. Gavrilenko, R. Taranko, V.K. Fedyanin, Phys. Status Solidi B 155, 489 (1989).

[19] Quin-feng Sun, Thung-han Lin, J. Phys., Condens. Matter 9, 4281 (1997); Eur. Phys. J. B 5, 913 (1998).

[20] E.R. Behringer, D.R. Anderson, B.H. Cooper, J.B. Marston, Phys. Rev. B 54, 14765 (1996).

[21] H. Nakanishi, H. Kasai, A. Okiji, Surf. Sci. 197, 595 (1998).

[22] G.A. Kimmel, B.H. Cooper, Phys. Rev. B 48, 12164 (1993).

[23] A.V. Onufriev, J.B. Marston, Phys. Rev. B 53, 13340 (1996). 